\title{
Evaluation of fecal pollution in coastal Italian waters by immunofluorescence
}

\author{
Renata Zaccone, Ermanno Crisafi, Gabriella Caruso \\ Istituto Sperimentale Talassografico CNR, Spianata S. Raineri, I-98122 Messina, Italy
}

\begin{abstract}
Direct detection of fecal coliforms as indicators of fecal pollution may assess water quality more accurately than culture methods. An immunochemical technique involving the use of specific antibodies was applied in addition to plate counts to evaluate Escherichia coli in seawater samples from different coastal areas in southern Italy. The immunofluorescent method showed high specificity for enteropathogenic $E$. coli although the presence of a threshold value of $10^{2}$ cell/100 $\mathrm{ml}$ sets a lower limit to the possibility of applying this method. It proved to be a rapid screening method to distinguish between polluted and unpolluted areas.
\end{abstract}

KEY WORDS: Monitoring $\cdot$ Escherichia cols $\cdot$ Marine pollution $\cdot$ Immunofluorescence

\section{INTRODUCTION}

Rapid enumeration of indicator organisms or pathogens in the aquatic environment is essential to assess water quality. The choice of Escherichia coli as an indicator organism of fecal pollution is accepted by various world organizations (OMS 1977, APHA 1985).

The methods officially accepted for quantification of $E$. coli have distinct limitations, due to a reliance on culture techniques. Samples must be treated in equipped laboratories within a short time from their collection; moreover processing times are usually rather long. Researchers therefore often reduce the number of analyses or divide them into groups.

It has also been shown that some 'injured' bacteria are unable to grow on standard culture media. In particular, fecal bacteria introduced in oligotrophic environments can develop various survival mechanisms They become nonculturable but remain viable up to 2 or $3 \mathrm{wk}$ in the environment (Brettar \& Hofle 1992, Islam et al. 1993); subsequently, they can be resuscitated to a culturable state (Nilsson et al. 1991). Traditional culture methods are inadequate to detect these stressed microorganisms. The condition of 'nonculturability' is different from 'nonviability'; Roszak \& Colwell (1987) have suggested the term of 'somnicell' for those cells which possess some vital attributes, but are unable to reproduce on culture media.

Direct microscopical counts by acridine orange or DAPI are not specific for individual species, whereas the use of fluorescent antibody methods or molecular probes can certainly provide more reliable information about the real presence of pathogenic bacteria, as well as about fecal indicators in the sea.

Direct and indirect immunofluorescence techniques have been used to quantify the presence of both pathogenic bacteria in the environment (Desmonts et al. 1990, Islam et al. 1993) and forms used as indicators of pollution (Munro \& Bianchi 1986). Such methods allow a remarkable rapidity of execution and the possibility of preserving the fixed samples up to 2 mo from the sampling date (Zaccone et al. 1990).

In this paper the results of a 4 yr survey are presented and discussed in order to evaluate the application of immunofluorescence for E. coli quantification in marine waters, in comparison with the membrane filter technique. The research was undertaken within the general framework of the 'Progetto Strategico per il monitoraggio automatico dell'inquinamento marino nel Mezzogiorno d'Italia' supported by CNR. It aims at developing new technologies and techniques with regard to the monitoring of sea pollution. 


\section{MATERIALS AND METHODS}

Sample collection. During oceanographic surveys carried out from 1988 to 1991, water samples from different coastal areas in Sicily and in Calabria, as well as from the brackish lake of Ganzirri, were collected in sterile polycarbonate bottles. A total of 122 samples were analysed to compare the immunological method with the conventional microbiological method. From each sample an aliquot was fixed in formalin ( $2 \%$ final) and preserved in the dark at $5^{\circ} \mathrm{C}$ to carry out the analyses by immunofluorescence (IF).

Plate counts. For culture analysis 10 and $100 \mathrm{ml}$ of each sample in 2 replicates were filtered through $0.45 \mu \mathrm{m}$ pore size Nuclepore filters, placed on the medium m-FC broth (Difco) supported by adsorbent pads (Nuclepore) and incubated at $44.5^{\circ} \mathrm{C}$ for the determination of fecal coliforms (FC).

Immunofluorescence counts. For direct immunofluorescent staining, the Behring sera 'Coli test serum pool A, B and C' conjugated with fluorescein-isothiocyanate were used. They were produced for the determination of enteropathogenic strains of $E$. coli. Sera are directed against the following serotypes: pool $A$ is specific against $E$. coli O26, O55, O111, O128; pool B against O86, O119, O125, O126, O127; pool C against O114, O142, O158.

Sera selectivity was tested by immunofluorescent staining with control strains ( $E$. coli $\mathrm{O} 26$ from culture collection Sclavo, and O-125), with 31 strains of environmental isolated $E$. coli, and with a group of different Gram-negative bacteria to assess cross-reactions. The degree of fluorescence was assessed on a scale ranging from $0+$ to $4+$ (see Table 1 ).

Following the analysis procedure reported by Munro \& Bianchi (1986) and Zaccone et al. (1990) subsample volumes ranging from 20 to $50 \mathrm{ml}$ were filtered on $0.2 \mu \mathrm{m}$ black polycarbonate membranes (Nuclepore) and washed with phosphate buffered saline (PBS): the filters were covered with hydrolyzed gelatine $(2 \%$ final) for $20 \mathrm{~min}$. After 3 washes with $5 \mathrm{ml}$ PBS the filters were stained with $1 \mathrm{ml}$ of sera diluted 1:10 in PBS for $30 \mathrm{~min}$. After 1 last rinse with PBS, filters were air dried and mounted with FA mounting fluid (Difco).

Observation by Zeiss fluorescent microscope (BP filter 450-490, FT 510, LP 520) allowed the enumeration of all bacterial cells that showed clear and fluorescent outlines, similar to control cells. The number of counted fields depended on the amount of cells on the filter. Twenty fields per slide were usually enumerated 'at random', but if no $E$. coli were observed, the count was extended up to 50 fields to establish that a result was negative.

Statistical tests. Samples positive with IF and plate methods were submitted to statistical analysis. The first step of the procedure was to examine the assumption of homogeneity of the counts. On microscopical and plate counts the following statistical parameters were calculated: average value, variance, standard error, and Fisher's dispersion index $\left(D^{2}\right)$. This procedure aimed at attributing the data to the Poisson distribution according to the formula: $D^{2}=(n-1) \mathrm{s}^{2} / X$, where $D^{2}$ was the dispersion index referred to a theoretical $\chi^{2}$ distribution with $(n-1)$ degrees of freedom; $n=$ number of data; $s^{2}=$ variance; $x=$ average value of the data. Values of $D^{2}$ higher than tabulated $\chi^{2}$ suggest that the Poisson distribution is not an adequate model of distribution and there is no homogeneity in bacterial counts. Values of $D^{2}$ lower than $\chi^{2}$ show that the Poisson distribution is an adequate model to explain the uniform distribution in each count (El-Shaarawi et al. 1981).

In order to study the range of the variations of cellular density values, we also examined how the coefficient of variation (CV), calculated for each filter, varied with the different mean counts. The $\mathrm{CV}$ expresses data dispersion compared to the arithmetic mean $(\mathrm{CV}=s / \mathrm{x})$ where $s=$ standard deviation and $x=$ arithmetic mean of the data (Kirchman et al. 1982). The error rate between the 2 methods was also calculated as $E \%=$ $(\mathrm{IF}-\mathrm{FC}) / \mathrm{FC}$

\section{RESULTS AND DISCUSSION}

Preliminary studies concerning the different study areas have been previously reported (Zaccone et al. 1990, Zaccone et al. 1991). They showed that IF values were higher than plate counts when $E$. coli counts were higher than $10^{2} / 100 \mathrm{ml}$.

The results of the tests for specificity of conjugated sera are shown in Table 1 in comparison with a 'home made' antiserum against $E$. coli ( $\mathrm{C} 2$ strain). The Behring sera cross-reacted with $32 \%$ of environmental isolated $E$. coli strains, even though weak reactions were observed with Salmonella and Serratia; no reaction was observed with marine bacteria. The $\mathrm{C} 2$ antiserum cross-reacted with a high number of $E$. coli strains $(60 \%)$. The first results of counts on seawater samples showed a good correlation between the 2 sera (Crisafi et al. 1994). Concerning the possibility of improving the sera specificity, Levasseur et al. (1992) studied the production of monoclonal antibodies for a rapid detection of Enterobacteriaceae, which crossreacted with all enterobacteria, but did not react with other Gram-negative strains.

Bacterial densities obtained by IF and plate methods were reported in Tables $2 \& 3$ with the mean values per study site. The calculation of Fisher's dispersion index $\left(D^{2}\right)$ showed a homogenous distribution on filters and plates; only a few samples showed values higher than 
Table 1. Results of cross-reaction tests by immunofluorescence with Behring and C2 antisera. Number of ' + ' represents the degree of fluorescence

\begin{tabular}{|c|c|c|c|c|c|}
\hline \multirow[t]{2}{*}{ Strain } & \multicolumn{2}{|c|}{ E. coli antisera } & \multirow[t]{2}{*}{ Strain } & \multicolumn{2}{|c|}{ E. coli antisera } \\
\hline & $A+B+C$ & $\mathrm{C} 2$ & & $A+B+C$ & $\mathrm{C} 2$ \\
\hline E. coli O26 & ++++ & +--- & C118 & ++-- & +--- \\
\hline E. coli 0125 & +++- & ++-- & C119 & ++-- & +++- \\
\hline E. coli $\mathrm{C} 2$ & ++-- & $+++t$ & $\mathrm{C} 122$ & ---- & +--- \\
\hline Salmonella enteritidis & +--- & ++++ & C 124 & ++-- & ++-- \\
\hline S. bovismorbificans & ---- & ++++ & C125 & +--- & --- \\
\hline S. panama & +--- & ++-- & C126 & ---- & ++-- \\
\hline S. paratyphi B & +--- & +++- & C127 & +--- & +--- \\
\hline S. typhi & +--- & ++++ & C130 & ---- & +--- \\
\hline S. virchow & ---- & $+t+t$ & C131 & ---- & +++- \\
\hline S. blockley & ++-- & +--- & $\mathrm{C} 142$ & ++-- & $++t-$ \\
\hline S. gallinarum & ---- & +--- & C144 & ++-- & +++- \\
\hline S. typhimurium & +--- & ++-- & $\mathrm{C} 145$ & ---- & +++- \\
\hline Shigella sonnei & ---- & ++-- & C150 & ---- & $++t-$ \\
\hline Sh. flexneri & ---- & ++-- & C151 & +--- & $+t+-$ \\
\hline Serratia marcescens & ++-- & ++-- & C152 & +--- & ---- \\
\hline Hafnia sp. & ---- & +--- & C153 & ++-- & $+t+t$ \\
\hline Y. enterocolitica $\mathrm{O} 3$ & ---- & +--- & C154 & ---- & ++++ \\
\hline Enterobacter & +--- & +-- & C155 & +--- & +++- \\
\hline Proteus vulgaris & $-\ldots-$ & ---- & C.158 & ---- & ---- \\
\hline Pr. rettgeri & ---- & ---- & C159 & ---- & ++-- \\
\hline Citrobacter sp. & ---- & ---- & C160 & +--- & +++- \\
\hline Enterococcus sp. & ---- & ---- & C161 & ---- & +++- \\
\hline Pseudomonas aeruginosa & ---- & +--- & $\mathrm{C} 162$ & $-\cdots--$ & ---- \\
\hline Pseudomonas $\mathrm{sp}$ & ---- & ---- & $\mathrm{C} 164$ & ++-- & +--- \\
\hline Vibrio cholerae non $\mathrm{O} 1$ & ---- & ---- & $\mathrm{C} 167$ & ---- & $--\ldots$ \\
\hline$V$. parahaemolyticus & ---- & ---- & $\mathrm{C} 168$ & ---- & ++-- \\
\hline$V$ alginolyticus & ---- & +--- & C175 & ---- & ---- \\
\hline Vibrio sp. & ---- & ---- & $\mathrm{C} 179$ & ++-- & +++- \\
\hline Flavobacterium sp. & ---- & ---- & C180 & +++- & ++-- \\
\hline Nitrosococcus oceanus & ---- & ---- & C192 & ---- & +--- \\
\hline
\end{tabular}

$\chi^{2}$. Only samples from the area of Calabria in 1990 were not homogenous by microscopic counts, whereas no homogeneity was found among the areas by plate counts. The significant values of $D^{2}$ per area are due to the extreme variability of CFU values.

The distribution of microscopical counts in relation to a theoretical curve of the $\mathrm{CV}$ is shown in Fig. 1. The variation in bacterial distribution per field appeared to be quite large. The CV value increased when microscopical counts were lower than 1 cell per field. Thus, the limit to the IF count method application was given by the low amount of coliforms in some samples.

In Fig. 2 the CVs calculated for plate counts are shown; they always express a presumptive count of fecal coliforms, since some strains of Citrobacter sp. and Klebsiella sp. can grow on medium. The mean values of $F C$ departed from the expected curve more than microscopic counts. According to Kirchman et al. (1982) if the data were consistent with Poisson distribution the points should fall equally on both sides of the line.
The 2 methods were compared by calculating the error rate and the results are shown in Fig. 3 . When the fecal coliforms values were lower than $10^{2} \mathrm{CFU} /$ $100 \mathrm{ml}$, the error rate was higher; whereas for FC values exceeding $10^{2} / 100 \mathrm{ml}$ the error rate was below $10 \%$. A sensitivity threshold of $10^{3}$ cells $\mathrm{ml}^{-1}$ was found by Desmonts et al. (1990) in detecting Salmonella spp. in chlorinated wastewater.

As expected, there was a significant difference between the 2 methods, since they evaluated different populations. The difference would support the hypothesis put forward by some authors (e.g. Roszack \& Colwell 1987) according to which direct count can reveal still viable, but noncultivable bacterial forms.

\section{CONCLUSION}

This paper confirmed our preliminary findings (Zaccone et al. 1990, 1991). The 2 methods evaluate 2 noncomparable populations with the counts by immunofluorescence higher than the counts on culture media 
Table 2. Cellular density, mean per field $(x)$, degree of freedom $(n-1)$, variance $\left(s^{2}\right)$, standard error $(\mathrm{SE})$, dispersion index $\left(D^{2}\right)$, critical chi-square $\left(\chi^{2}\right)$ and coefficient of variation $(\mathrm{CV})$ for each sample and per area by immunofluorescent assay. "Significant $D^{2}$ value

\begin{tabular}{|c|c|c|c|c|c|c|c|c|c|}
\hline & Sample & Cell $\mathrm{ml}^{-1}$ & $x$ & $n-1$ & $s^{2}$ & $\mathrm{SE}$ & $D^{2}$ & $\chi^{2}$ & CV \\
\hline $\begin{array}{l}\text { Strait of } \\
\text { Messina }\end{array}$ & $\begin{array}{l}\text { S1 } \\
\text { S2 } \\
\text { S3 } \\
\text { S4 } \\
\text { S5 } \\
\text { S6 } \\
\text { S7 } \\
\text { S8 } \\
\text { S9 } \\
\text { S10 } \\
\text { S11 } \\
\text { S12 } \\
\text { S13 } \\
\text { Area }\end{array}$ & $\begin{array}{r}617.59 \\
574.99 \\
2683.27 \\
511.10 \\
217.22 \\
293.88 \\
115.00 \\
191.66 \\
63.89 \\
2555.50 \\
42.59 \\
766.65 \\
159.72 \\
681.29\end{array}$ & $\begin{array}{l}2.42 \\
2.25 \\
1.75 \\
2 \\
1.42 \\
1.91 \\
0.75 \\
0.75 \\
0.42 \\
1.67 \\
0.17 \\
1.5 \\
0.42 \\
1.34\end{array}$ & $\begin{array}{r}23 \\
23 \\
23 \\
23 \\
23 \\
23 \\
23 \\
23 \\
23 \\
23 \\
23 \\
23 \\
23 \\
311\end{array}$ & $\begin{array}{l}4.41 \\
4.69 \\
0.52 \\
2.67 \\
1.91 \\
4.91 \\
0.85 \\
0.52 \\
0.58 \\
2.72 \\
0.14 \\
1.08 \\
0.41 \\
2.04\end{array}$ & $\begin{array}{l}0.43 \\
0.44 \\
0.15 \\
0.33 \\
0.28 \\
0.45 \\
0.19 \\
0.15 \\
0.16 \\
0.34 \\
0.08 \\
0.21 \\
0.13 \\
0.08\end{array}$ & $\begin{array}{r}22.93 \\
27.47 \\
6.85 \\
30.67 \\
31 \\
38.92 \\
26.19 \\
15.97 \\
31.82 \\
37.57 \\
19.17 \\
16.61 \\
22.62 \\
474.67\end{array}$ & $\begin{array}{r}41.64 \\
41.64 \\
41.64 \\
41.64 \\
41.64 \\
41.64 \\
41.64 \\
41.64 \\
41.64 \\
41.64 \\
41.64 \\
41.64 \\
41.64 \\
629.66\end{array}$ & $\begin{array}{l}0.87 \\
0.96 \\
0.41 \\
0.82 \\
0.97 \\
1.16 \\
1.23 \\
0.96 \\
1.82 \\
0.98 \\
2.24 \\
0.7 \\
1.54 \\
1.07\end{array}$ \\
\hline $\begin{array}{l}\text { Augusta } \\
\text { Bay }\end{array}$ & $\begin{array}{l}\text { A1 } \\
\text { A2 } \\
\text { A3 } \\
\text { A4 } \\
\text { A5 } \\
\text { A6 } \\
\text { A7 } \\
\text { A8 } \\
\text { A9 } \\
\text { A10 } \\
\text { A11 } \\
\text { A12 } \\
\text { A13 } \\
\text { A14 } \\
\text { A15 } \\
\text { Area }\end{array}$ & $\begin{array}{r}107.33 \\
245.33 \\
61.33 \\
178.88 \\
61.33 \\
153.33 \\
92.00 \\
46.00 \\
199.33 \\
171.73 \\
233.06 \\
15.33 \\
107.33 \\
260.66 \\
153.33 \\
139.09\end{array}$ & $\begin{array}{l}0.7 \\
1.6 \\
0.4 \\
0.7 \\
0.4 \\
1 \\
0.6 \\
0.3 \\
1.3 \\
1.12 \\
1.52 \\
0.1 \\
0.7 \\
1.7 \\
1 \\
0.9\end{array}$ & $\begin{array}{r}19 \\
19 \\
19 \\
19 \\
19 \\
19 \\
19 \\
19 \\
19 \\
24 \\
24 \\
19 \\
19 \\
19 \\
19 \\
309\end{array}$ & $\begin{array}{l}0.61 \\
2.04 \\
0.44 \\
0.61 \\
0.84 \\
1.8 \\
0.44 \\
0.21 \\
0.61 \\
2.43 \\
2.09 \\
0.09 \\
1.41 \\
3.61 \\
1 \\
1.48\end{array}$ & $\begin{array}{l}0.17 \\
0.32 \\
0.15 \\
0.17 \\
0.21 \\
0.30 \\
0.15 \\
0.10 \\
0.17 \\
0.31 \\
0.29 \\
0.07 \\
0.27 \\
0.42 \\
0.22 \\
0.07\end{array}$ & $\begin{array}{l}16.56 \\
24.23 \\
20.9 \\
16.56 \\
39.9^{\circ} \\
34.2 \\
13.93 \\
13.3 \\
8.92 \\
51.98^{\circ} \\
32.99 \\
17.1 \\
38.27^{\circ} \\
40.35^{\circ} \\
19 \\
513.06\end{array}$ & $\begin{array}{r}36.19 \\
36.19 \\
36.19 \\
36.19 \\
36.19 \\
36.19 \\
36.19 \\
36.19 \\
36.19 \\
42.98 \\
42.98 \\
36.19 \\
36.19 \\
36.19 \\
36.19 \\
622.00\end{array}$ & $\begin{array}{l}1.11 \\
0.89 \\
1.66 \\
1.11 \\
2.29 \\
1.34 \\
1.1 \\
1.53 \\
0.6 \\
1.39 \\
0.95 \\
3 \\
1.7 \\
1.12 \\
1 \\
1.36\end{array}$ \\
\hline $\begin{array}{l}\text { Ganzirri } \\
\text { Lake }\end{array}$ & $\begin{array}{c}\text { G1 } \\
\text { G2 } \\
\text { G3 } \\
\text { G4 } \\
\text { G5 } \\
\text { G6 } \\
\text { G7 } \\
\text { Area }\end{array}$ & $\begin{array}{r}85.86 \\
76.66 \\
73.59 \\
32.51 \\
100.80 \\
75.60 \\
83.16 \\
75.45\end{array}$ & $\begin{array}{l}0.6 \\
0.5 \\
0.5 \\
0.3 \\
0.8 \\
0.6 \\
1.1 \\
0.6\end{array}$ & $\begin{array}{r}24 \\
19 \\
24 \\
30 \\
19 \\
19 \\
19 \\
160\end{array}$ & $\begin{array}{l}0.4 \\
0.45 \\
0.73 \\
0.19 \\
0.56 \\
0.44 \\
0.49 \\
0.52\end{array}$ & $\begin{array}{l}0.13 \\
0.15 \\
0.17 \\
0.08 \\
0.17 \\
0.15 \\
0.16 \\
0.06\end{array}$ & $\begin{array}{c}17.41 \\
17.1 \\
36.48 \\
22.25 \\
13.3 \\
13.93 \\
8.46 \\
141.48\end{array}$ & $\begin{array}{r}42.98 \\
36.19 \\
42.98 \\
50.89 \\
36.19 \\
36.19 \\
36.19 \\
275.39\end{array}$ & $\begin{array}{l}1.14 \\
1.34 \\
1.78 \\
1.69 \\
0.93 \\
1.1 \\
0.64 \\
1.23\end{array}$ \\
\hline $\begin{array}{l}\text { Calabrian } \\
\text { coast } 1990\end{array}$ & $\begin{array}{l}\text { C1 } \\
\text { C2 } \\
\text { C3 } \\
\text { C4 } \\
\text { C5 } \\
\text { C6 } \\
\text { C7 } \\
\text { C8 } \\
\text { C9 } \\
\text { C10 } \\
\text { C11 } \\
\text { C12 } \\
\text { C13 } \\
\text { Area }\end{array}$ & $\begin{array}{r}3275.9 \\
403.19 \\
75.60 \\
113.40 \\
52.92 \\
15.12 \\
120.96 \\
151.19 \\
98.28 \\
143.63 \\
302.39 \\
68.04 \\
25.20 \\
130.83\end{array}$ & $\begin{array}{l}26 \\
3.2 \\
0.6 \\
1.5 \\
0.7 \\
0.2 \\
1.6 \\
1.2 \\
1.3 \\
1.9 \\
2.4 \\
0.9 \\
0.2 \\
2.3\end{array}$ & $\begin{array}{r}19 \\
19 \\
19 \\
19 \\
19 \\
19 \\
19 \\
19 \\
19 \\
19 \\
19 \\
19 \\
19 \\
249\end{array}$ & $\begin{array}{c}62.4 \\
1.16 \\
0.64 \\
2.25 \\
0.61 \\
0.16 \\
1.48 \\
0.76 \\
1.21 \\
0.69 \\
0.44 \\
0.49 \\
0.16 \\
27.42\end{array}$ & $\begin{array}{l}2.50 \\
0.24 \\
0.36 \\
0.34 \\
0.17 \\
0.09 \\
0.27 \\
0.19 \\
0.25 \\
0.19 \\
0.15 \\
0.16 \\
0.09 \\
0.33\end{array}$ & $\begin{array}{c}45.6^{\circ} \\
6.89 \\
20.26 \\
28 \\
16.56 \\
15.2 \\
19.47 \\
12.03 \\
17.68 \\
6.9 \\
3.48 \\
10.34 \\
15.2 \\
2974.2\end{array}$ & $\begin{array}{r}36.19 \\
36.19 \\
36.19 \\
36.19 \\
36.19 \\
36.19 \\
36.19 \\
36.19 \\
36.19 \\
36.19 \\
36.19 \\
36.19 \\
36.19 \\
482.43\end{array}$ & $\begin{array}{l}0.31 \\
0.34 \\
2.68 \\
1 \\
1.11 \\
2 \\
0.76 \\
0.73 \\
0.85 \\
0.44 \\
0.28 \\
0.7 \\
2 \\
2.28\end{array}$ \\
\hline $\begin{array}{l}\text { Calabrian } \\
\text { coast } 1991\end{array}$ & $\begin{array}{l}\text { C14 } \\
\text { C15 } \\
\text { C16 } \\
\text { C17 } \\
\text { C18 } \\
\text { C19 } \\
\text { C20 } \\
\text { C21 } \\
\text { C22 } \\
\text { C23 } \\
\text { C24 } \\
\text { C25 } \\
\text { C26 } \\
\text { C27 } \\
\text { C28 } \\
\text { C29 } \\
\text { C } 30 \\
\text { C } 31 \\
\text { Area }\end{array}$ & $\begin{array}{r}15.12 \\
182.69 \\
15.12 \\
68.04 \\
15.12 \\
52.92 \\
90.72 \\
211.67 \\
45.36 \\
25.20 \\
7.56 \\
26.46 \\
98.28 \\
30.24 \\
37.80 \\
15.12 \\
22.68 \\
56.70 \\
58.92\end{array}$ & $\begin{array}{l}0.2 \\
2.5 \\
0.2 \\
0.9 \\
0.1 \\
0.7 \\
1.2 \\
2.8 \\
0.6 \\
0.2 \\
0.1 \\
0.4 \\
1.3 \\
0.4 \\
0.5 \\
0.2 \\
0.3 \\
0.8 \\
0.7\end{array}$ & $\begin{array}{l}19 \\
19 \\
19 \\
19 \\
19 \\
19 \\
19 \\
19 \\
19 \\
19 \\
19 \\
19 \\
19 \\
19 \\
19 \\
19 \\
19 \\
19 \\
359\end{array}$ & $\begin{array}{l}0.16 \\
1.65 \\
0.36 \\
1.69 \\
0.09 \\
1.01 \\
1.76 \\
2.36 \\
0.24 \\
0.16 \\
0.09 \\
0.43 \\
1.71 \\
0.24 \\
0.45 \\
0.16 \\
0.21 \\
0.69 \\
1.33\end{array}$ & $\begin{array}{l}0.09 \\
0.29 \\
0.13 \\
0.29 \\
0.07 \\
0.22 \\
0.30 \\
0.34 \\
0.11 \\
0.09 \\
0.07 \\
0.15 \\
0.29 \\
0.11 \\
0.15 \\
0.09 \\
0.10 \\
0.19 \\
0.06\end{array}$ & $\begin{array}{l}15.2 \\
12.54 \\
34 \\
35.67 \\
17.1 \\
27.41 \\
27.9 \\
16.02 \\
7.6 \\
15.2 \\
17.1 \\
23.21 \\
24.99 \\
11.4 \\
17.1 \\
15.2 \\
13.3 \\
17.42 \\
644.38\end{array}$ & $\begin{array}{r}36.19 \\
36.19 \\
36.19 \\
36.19 \\
36.19 \\
36.19 \\
36.19 \\
36.19 \\
36.19 \\
36.19 \\
36.19 \\
36.19 \\
36.19 \\
36.19 \\
36.19 \\
36.19 \\
36.19 \\
36.19 \\
738.32\end{array}$ & $\begin{array}{l}2 \\
0.49 \\
3 \\
1.44 \\
3 \\
1.43 \\
1.1 \\
0.55 \\
0.82 \\
2 \\
3 \\
1.87 \\
1.01 \\
1.22 \\
1.34 \\
2 \\
1.53 \\
1.1 \\
1.56\end{array}$ \\
\hline
\end{tabular}


Table 3. Cellular density, mean per plate $(x)$, degree of freedom $(n-1)$, variance $\left(s^{2}\right)$, standard error (SE), dispersion index $\left(D^{2}\right)$, critical chi-square $\left(\chi^{2}\right)$ and coefficient of variation for each sample and per area by plate count. $\cdot$ Significant $D^{2}$ value

\begin{tabular}{|c|c|c|c|c|c|c|c|c|c|}
\hline & Sample & $\mathrm{CFU} / 100 \mathrm{ml}$ & $x$ & $n-1$ & $s^{2}$ & SE & $D^{2}$ & $x^{2}$ & $C V$ \\
\hline Strait of & S1 & 845 & 84.50 & 1 & 72.25 & 6.01 & 0.85 & 6.63 & 0.10 \\
\hline \multirow[t]{8}{*}{ Messina } & S2 & 25600 & 256.00 & 1 & 576 & 16.97 & 2.25 & 6.63 & 0.09 \\
\hline & $\mathrm{S} 4$ & 11 & 247 & 1 & 225 & 10.61 & 0.91 & 6.63 & 0.06 \\
\hline & S5 & 11 & 10.50 & 1 & 0.25 & 0.35 & 0.02 & 6.63 & 0.05 \\
\hline & S6 & 9 & 10.50 & 1 & 0.25 & 0.35 & 0.02 & 6.63 & 0.05 \\
\hline & $\mathrm{S} 7$ & 5 & 9.00 & 1 & 0 & 0.00 & 0.00 & 6.63 & 0.00 \\
\hline & $\mathrm{S} 8$ & 23 & 0.50 & 1 & 0.25 & 0.35 & 0.50 & 6.63 & 1.00 \\
\hline & S9 & 20 & 23.00 & 1 & 1 & 0.71 & 0.04 & 6.63 & 0.04 \\
\hline & Area & 2947 & 80.13 & 15 & 10504 & 25.62 & $1966.40^{\circ}$ & 30.88 & 1.28 \\
\hline Augusta & A1 & 3 & 3.00 & 1 & 0 & 0.00 & 0.00 & 6.63 & 0.00 \\
\hline \multirow[t]{15}{*}{ Bay } & $\mathrm{A} 2$ & 92 & 91.50 & 1 & 30.25 & 3.89 & 0.33 & 6.63 & 0.06 \\
\hline & $\mathrm{A} 3$ & 1 & 1.00 & 1 & 0 & 0.00 & 0.00 & 6.63 & 0.00 \\
\hline & A4 & 135 & 134.50 & 1 & 42.25 & 4.60 & 0.31 & 6.63 & 0.05 \\
\hline & A5 & 2 & 1.50 & 1 & 2.25 & 1.06 & 1.50 & 6.63 & 1.00 \\
\hline & A6 & 132 & 132.00 & 1 & 289 & 12.02 & 2.19 & 6.63 & 0.13 \\
\hline & A7 & 24 & 23.50 & 1 & 2.25 & 1.06 & 0.09 & 6.63 & 0.06 \\
\hline & A8 8 & 7 & 7.00 & 1 & 25 & 3.53 & 3.57 & 6.63 & 0.71 \\
\hline & A9 & 20 & 20.00 & 1 & 36 & 4.24 & 1.80 & 6.63 & 0.30 \\
\hline & A. 10 & 8 & 7.50 & 1 & 0.25 & 0.35 & 0.03 & 6.63 & 0.07 \\
\hline & A11 & 13 & 13.00 & 1 & 36 & 4.24 & 2.77 & 6.63 & 0.00 \\
\hline & A 12 & 142 & 142.00 & 1 & 0 & 0.00 & 0.00 & 6.63 & 0.06 \\
\hline & A13 & 235 & 235.00 & 1 & 225 & 10.61 & 0.96 & 6.63 & 0.00 \\
\hline & A14 & 2 & 2.00 & 1 & 0 & 0.00 & 0.00 & 6.63 & 0.00 \\
\hline & A.15 & 6 & 6.00 & 1 & 0 & 0.00 & 0.00 & 6.63 & 0.00 \\
\hline & Area & 55 & 54.60 & $2 \hat{9}$ & 5098.03 & 13.04 & $2706.09^{\circ}$ & 52.34 & 1.31 \\
\hline Ganzirri & G1 & 208 & 207.50 & 1 & 1806.25 & 30.05 & $8.70^{\circ}$ & 6.63 & 0.20 \\
\hline \multirow[t]{8}{*}{ Lake } & $\mathrm{G} 2$ & 9 & 8.50 & 1 & 2.25 & 1.06 & 0.26 & 6.63 & 0.18 \\
\hline & G3 & 53 & 53.00 & 1 & 36 & 4.24 & 0.68 & 6.63 & 0.11 \\
\hline & $\mathrm{G} 4$ & 140 & 14.00 & 1 & 1 & 0.71 & 0.07 & 6.63 & 0.07 \\
\hline & G5 & 82 & 82.00 & 1 & 100 & 7.07 & 1.22 & 6.63 & 0.12 \\
\hline & G6 & 1380 & 138.00 & 1 & 0 & 0.00 & 0.00 & 6.63 & 0.00 \\
\hline & G7 & 820 & 82.00 & 1 & 0 & 0.00 & 0.00 & 6.63 & 0.00 \\
\hline & G8 & 110 & 11.00 & 1 & 4 & 1.41 & 0.36 & 6.63 & 0.18 \\
\hline & Area & 350 & 74.50 & 15 & 4536.75 & 16.84 & $913.44^{\circ}$ & 32.80 & 0.90 \\
\hline \multirow{14}{*}{$\begin{array}{l}\text { Calabrian } \\
\text { coast } 1990\end{array}$} & $\mathrm{C} 1$ & 50 & 0.50 & 1 & 0.25 & 0.35 & 0.50 & 6.63 & 1.00 \\
\hline & $\mathrm{C} 2$ & 1065 & 106.50 & 1 & 380.25 & 13.79 & 3.57 & 6.63 & 0.18 \\
\hline & C3 & 160 & 160.00 & 1 & 1 & 0.71 & 0.01 & 6.63 & 0.01 \\
\hline & $\mathrm{C} 4$ & 1015 & 101.50 & 1 & 2.25 & 1.06 & 0.02 & 6.63 & 0.01 \\
\hline & $\mathrm{C} 5$ & 1145 & 114.50 & 1 & 240.25 & 10.96 & 2.10 & 6.63 & 0.13 \\
\hline & C6 & 225 & 22.50 & 1. & 30.25 & 3.89 & 1.34 & 6.63 & 0.24 \\
\hline & $C 7$ & 900 & 9.00 & 1 & 0 & 0.00 & 0.00 & 6.63 & 0.00 \\
\hline & $\mathrm{C} 8$ & 235 & 23.50 & 1 & 6.25 & 1.77 & 0.26 & 6.63 & 0.11 \\
\hline & $\mathrm{C} 9$ & 105 & 10.50 & 1 & 0.25 & 0.35 & 0.02 & 6.63 & 0.05 \\
\hline & $\mathrm{C} 10$ & 250 & 2.50 & 1 & 6.25 & 1.77 & 2.50 & 6.63 & 1.00 \\
\hline & C11 & 75 & 7.50 & 1 & 56.25 & 5.30 & $7.50^{\circ}$ & 6.63 & 1.00 \\
\hline & C12 & 121 & 120.50 & 1 & & & & & \\
\hline & C13 & 85 & 8.50 & 1 & 2.25 & 1.06 & 0.26 & 6.63 & 0.18 \\
\hline & Area & 418 & 52.90 & 25 & 3187.64 & 11.07 & $1506.88^{\circ}$ & 46.93 & 1.07 \\
\hline \multirow{18}{*}{ coast 1991} & C14 & 575 & 57.80 & 1 & 132.25 & 8.13 & 2.30 & 6.63 & 0.20 \\
\hline & C15 & 900 & 9.00 & 1 & 49 & 4.95 & 5.40 & 6.63 & 0.70 \\
\hline & C16 & 150 & 15.00 & 1 & 25 & 3.3 .53 & 1.60 & 6.63 & 0.30 \\
\hline & C17 & 425 & 42.50 & 1 & 42.25 & 4.60 & 0.10 & 6.63 & 0.15 \\
\hline & C18 & 795 & 79.50 & $i$ & 42.25 & 4.60 & 0.50 & 6.63 & 0.08 \\
\hline & C19 & 850 & 8.50 & 1 & 0.25 & 0.35 & 0.03 & 6.63 & 0.06 \\
\hline & $\mathrm{C} 20$ & 1650 & 16.50 & 1 & 0.25 & 0.35 & 0.02 & 6.63 & 0.03 \\
\hline & C21 & 490 & 49.00 & 1 & 64 & 5.66 & 1.31 & 6.63 & 0.16 \\
\hline & C22 & 6950 & 69.50 & 1 & 2.25 & 1.06 & 0.03 & 6.63 & 0.21 \\
\hline & $\mathrm{C} 23$ & 4700 & 47.00 & 1 & $16 \overline{9}$ & 9.19 & 3.59 & 6.63 & 0.27 \\
\hline & $\mathrm{C} 24$ & 114 & 114.00 & 1 & 0 & 0.00 & 0.00 & 6.63 & 0.00 \\
\hline & C25 & 11850 & 118.50 & 1 & 20.25 & 3.18 & 0.17 & 6.63 & 0.04 \\
\hline & C26 & 5300 & 53.00 & 1 & 4 & 1.41 & 0.08 & 6.63 & 0.04 \\
\hline & $\mathrm{C} 27$ & 1600 & 16.00 & 1 & 1 & 0.71 & 0.06 & 6.63 & 0.06 \\
\hline & C28 & 141 & 14.00 & 1 & 4 & 1.41 & 0.30 & 6.63 & 0.14 \\
\hline & C29 & 265 & 26.50 & 1 & 2.25 & 1.06 & 0.08 & 6.63 & 0.05 \\
\hline & C31 & 595 & 59.50 & 1 & 12.25 & 2.47 & 0.20 & 6.63 & 0.06 \\
\hline & Area & 2075 & 46.80 & 33 & 1141.81 & 5.80 & $805.22^{\circ}$ & 53.67 & 0.72 \\
\hline
\end{tabular}




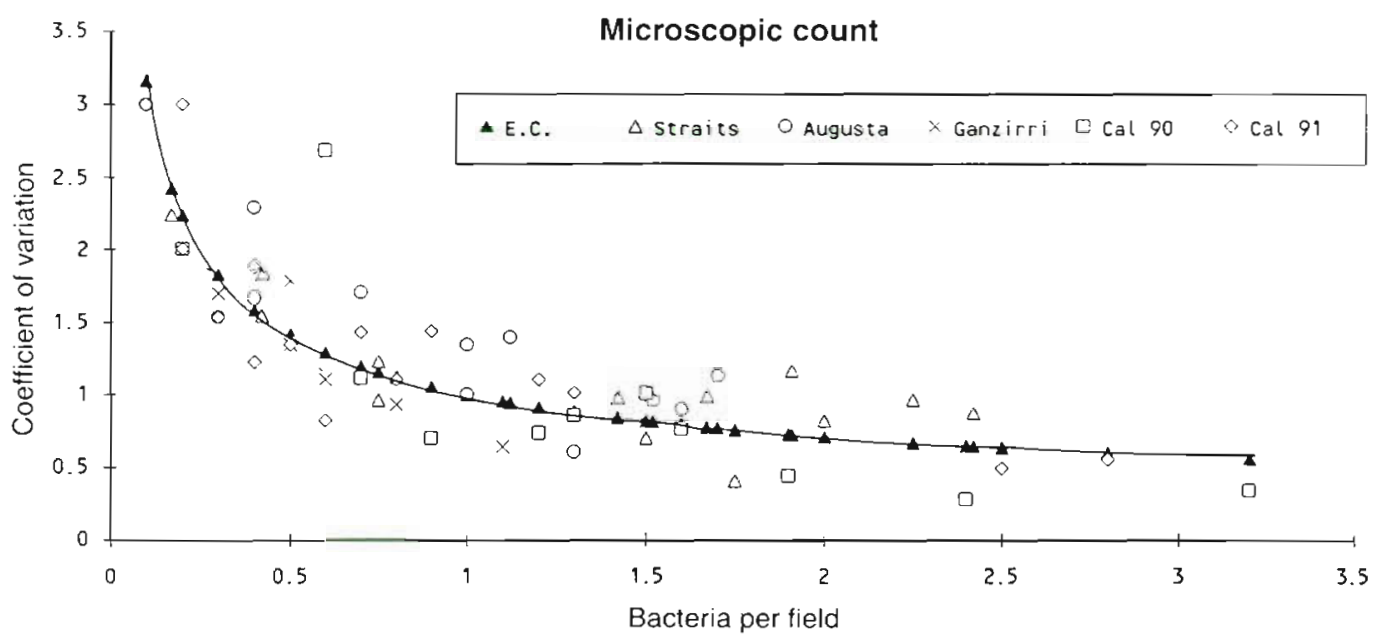

Fig. 1. Coefficient of variation for a mean number of bacteria per microscope field. (^) Expected curve (E.C.) from the Poisson distribution (CV $=1 / \sqrt{ } x\}$

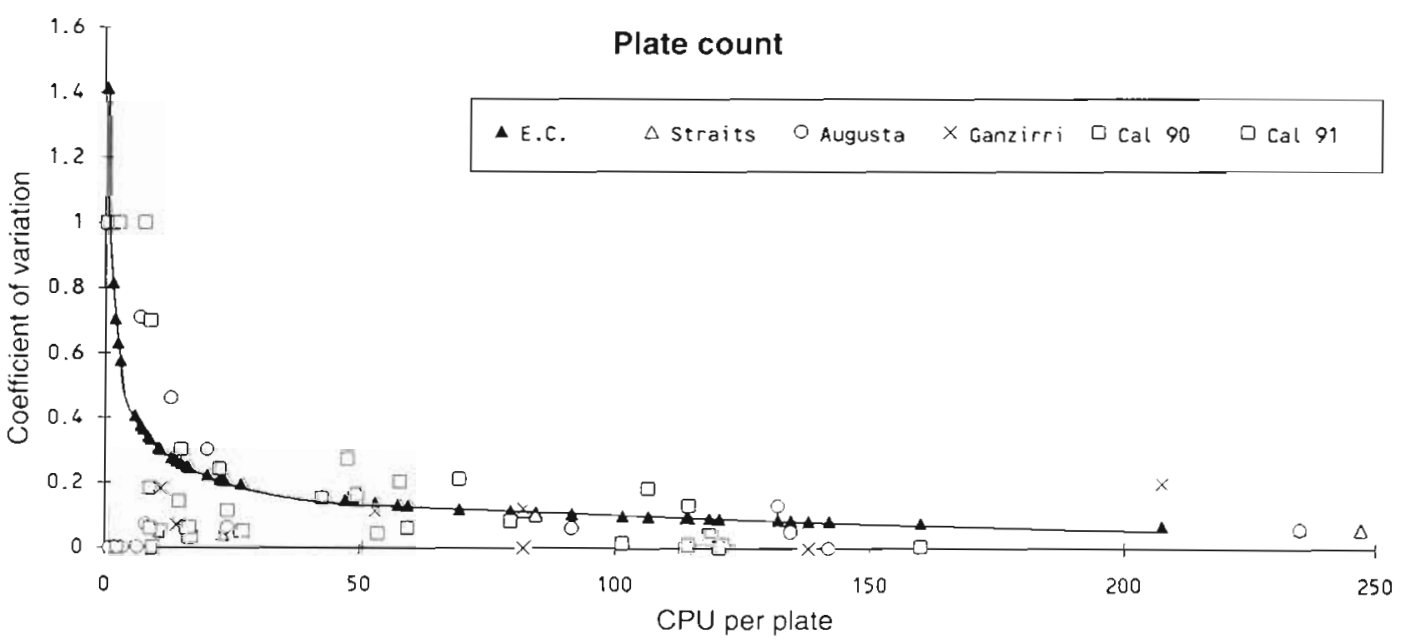

Fig. 2. Coefficient of variation for a mean number of CFU per plate. (4) Expected curve (E.C.) from the Poisson distribution $\left(\mathrm{CV}=1 / V_{X}\right)$

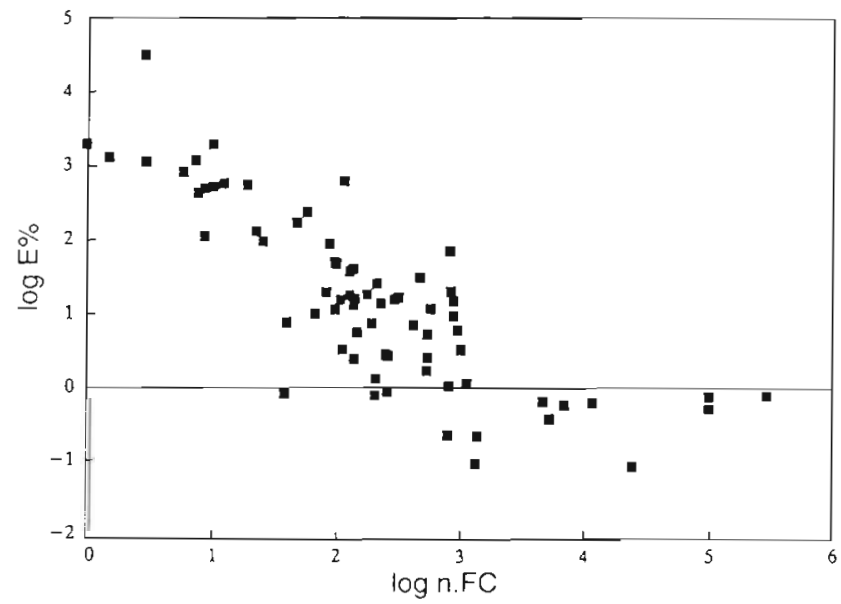

Fig 3. Error rate between fecal coliforms (FC) and immunofluorescence direct count (IF) calculated as $\mathrm{E} \%=(\mathrm{IF}-\mathrm{FC}) / \mathrm{FC}$ by 2 orders of magnitude. The application of immunofluorescent method for seawater showed qualitative and quantitative limits: qualitative limitations depended on the specificity of the serum directed against a limitated number of serotypes.

The existence of a threshold value below which the IF technique cannot be applied sets an inferior limit to the possibility of applying this method.

The limit of detection depended also on the significant amount of $E$. coli on samples. When specific bacteria were below $1 \%$ of the total population, a large amount of sample should be filtered, but high particle content prevented us from increasing the volume that could be filtered (Brettar \& Hofle 1992). In lightly polluted areas the probability of estimation errors is higher, whereas in polluted areas it can give more accurate results.

When used for environmental monitoring, this method is easy to carry out and suitable for a wide 
range of samples. It turned out to be a rapid screening to distinguish between polluted and unpolluted areas. Further studies will be necessary to perfect this technique; however, it has promise as a method that can be applied in continuous monitoring investigations by means of an automatic sampler, which will allow fixative automatic addition as well as timed remote sampling

Acknowledgements. This work was supported by the C.N.R.: 'Progetto Strategico per il monitoraggio automatico dell' inquinamento marino nel Mezzogiorno d'Italia'. The authors thank Dr Lucila Acosta Pomar for the suggestions given in the statistical elaboration and M. Maurizio Azzaro for technical assistance.

\section{LITERATURE CITED}

American Public Health Association (APHA) (1985) Standard methods for the examination of water and wastewater, 16th edn. American Public Health Association, Inc, Washington, $\mathrm{DC}$

Brettar I, Hofle M (1992) Influence of ecosystematic factors on survival of Escherichia coli after large-scale release into lake water mesocosms. Appl environ Microbiol 58: $2201-2210$

Crisafi E, Russo G, Oliveri S, Zaccone R (1994) Ricerca di $E$. coli e Candida in acque marine costiere mediante sieri immuni 'home made' In: Atti Workshop 'Il monitoraggio automatico dell'inquinamento marino, stato dell'arte e prospettive'. Taranto, 9-10 April 1992

Desmonts C, Minet J, Colwell RR, Cormier M (1990) Fluorescent-antibody method useful for detecting viable but nonculturable Salmonella spp. in chlorinated wastewater. Appl environ Microbiol 56:1448-1452

El-Shaarawi AH, Esterby SR, Dutka BJ (1981) Bacterial density in water determined by Poisson or negative binomial distributions. Appl environ Microbiol 41:107-116
Islam MS, Hasan MK, Miah MA, Sur GC, Felsenstein A Venkatesan M, Sack RB, Albert MJ (1993) Use of the polymerase chain reaction and fluorescent-antibody methods for detecting viable but nonculturable Shigella dysenteriae type 1 in laboratory microcosms. Appl environ Microbiol $59.536-540$

Kirchman D, Sigda J, Kapuscinski R, Mitchell R (1982) Statistical analysis of the direct count method for enumerating bacteria. Appl environ Microbiol 44:376-382

Levasseur S, Husson MO, Leitz R, Merlin F, Laurent F, Peladan F, Drocourt JL, Leclerc $H$, Van Hoegaerden $M$ (1992) Rapid detection of members of the family Enterobacteriaceae by a monoclonal antibody. Appl environ Microbiol 58:1524-1529

Munro P, Bianchi M (1986) Comparaison de méthodes directes (microscopiques) et indirectes (par mise en culture) dans l'évaluation d'une pollution bactérienne d'origine fécale étude préliminaire. In: GERBAM - 2ème Colloque International de Bactériologie marine-CNRS, Brest, 1-5 octobre 1984. Actes de Colloques, IFREMER 3: $515-520$

Nilsson L, Oliver JD, Kjelleberg S (1991) Resuscitation of Vibrio vulnificus from the viable but nonculturable state. J Bacteriol 173:5054-5059

Organisation Mondiale de la Santé (OMS) (1977) Directives applicables à la surveillance sanitaire de la qualité des eaux littorales. Organisation Mondiale de la Santé, Bureau régional de l'Europe, Copenhague

Roszack DB, Colwell RR (1987) Metabolic activity of bacterial cells enumerated by direct viable count. Appl environ Microbiol 53:2889-2893

Zaccone R, Caruso G, Crisafi E (1991) Utilisation d'anticorps fluorescents pour le comptage de $E$. coli en eau de mer. In: Actes du Gème Colloque International d'Océanographie Médicale, CERBOM, Revue Internationale d'Océanographie Médicale, 101-104:90-93

Zaccone R, La Ferla R, Genovese L, Crisafi E (1990) Primi dati sull utilizzazione di anticorpi fluorescenti per la ricerca di E. coli nell'ambiente marino. Oebalia (Suppl) XVI-2: 809-812 